\title{
Multi-materials with strong interface: Variational modelings
}

\author{
Anne Laure Bessoud ${ }^{\mathrm{a}}$, Françoise Krasucki ${ }^{\mathrm{b}}$ and Gérard Michaille ${ }^{\mathrm{c}}$ \\ a I3M, UMR-CNRS 5149 et LMGC, UMR-CNRS 5508, Université Montpellier 2, Case courier 048, \\ Place Eugène Bataillon, 34095 Montpellier Cedex 5, France \\ E-mail: bessoud@lmgc.univ-montp2.fr \\ b ACSIOM, I3M, UMR-CNRS 5149, Université Montpellier 2, Case courier 051, Place Eugène \\ Bataillon, 34095 Montpellier Cedex 5, France \\ E-mail: krasucki@math.univ-montp2.fr \\ ${ }^{\mathrm{c}}$ ACSIOM and AVA, I3M, UMR-CNRS 5149, Université Montpellier 2 et Université de Nîmes, \\ Case courier 051, Place Eugène Bataillon, 34095 Montpellier Cedex 5, France \\ E-mail:micha@math.univ-montp2.fr
}

\begin{abstract}
We introduce a simplified model for a multi-material made up of two elastic bodies connected by a strong thin material layer whose stiffness grows as $\frac{1}{\varepsilon}$. The model is obtained by identifying the $\Gamma$-limit of the stored strain energy functional of the physical problem when the thickness $\varepsilon$ of the intermediate layer tends to zero. The intermediate layer behaves as a stiffening elastic membrane. Furthermore, in the linear anisotropic case, we establish the strong convergence of the exact solution toward the solution of the limit problem.
\end{abstract}

Keywords: elasticity, multi-materials, multi-scaling, $\Gamma$-convergence

\section{Introduction}

In the problem of joining together two separate elastic bodies, an interesting question is the modeling of the behavior of the thin layer interposed between the adherents. In this problem, at least two parameters are important: one is geometrical and is associated with the intermediate layer thickness, the other one is material and associated with its stiffness. Let $\varepsilon$ be the magnitude order of the layer thickness with respect to a global linear dimension of the structure. Two extreme situations are worth noticing: (i) the stiffness of the constitutive material of the layer is of order $\varepsilon$ with respect to that of the adherents (we can speak of "gluing"); (ii) the stiffness of the constitutive material of the layer is of order $\frac{1}{\varepsilon}$ with respect to that of the adherents (we can speak of "welding"). These situations arise, for instance, in the study of composites and other fields of engineering. More recently, works about functionally graded materials pointed out that the differences in the thermal expansion coefficients of the component lead to the formation of residual stresses, which may be critical for the integrity of the composite. Functionally graded materials (FGM) are a new generation of engineered materials consisting of metallic and ceramic components whose use improves the properties of many technical devices. Engineers accomplish this by using reinforcements with different properties, sizes, and shapes, as well as by interchanging the roles 
of the reinforcement. Owing to the many variables that control the design of functionally graded microstructures, full exploitation of the FGM's potential requires the development of appropriate modeling strategies for their response to combined thermomechanical loads.

In a very simplified modeling such problem, from a mechanical point of view, can be reduced to the transmission of stresses between three elastic bodies where the intermediate body is a very thin layer. The computation of the solution $\mathbf{u}^{\varepsilon}$ is usually very difficult, even if numerical methods are employed. This is due to the fact that the thinness of the layer requires a fine mesh, which in turn implies an increase of the degrees of freedom of the system. Moreover, the differences in the elastic coefficients produce numerical instabilities in the stiffness matrix. A good way to overcome these difficulties is the introduction of a variational limit problem taking into account the magnitude orders of both thickness and material coefficients and whose solution $\mathbf{u}$ can be "easily" computed. In this limit problem, a "material surface" substitutes for the thin layer. This material surface carries a suitable surface energy density obtained through the limiting process from the global volume energy of the thin layer. Hence, the potential energy of the whole system is obtained adding the surface energy to the energies of the two others bodies.

The "gluing situation" has been deeply studied, starting from the pioneering work of [12], in the technical and mathematical literature. In the limit problem the "material surface" behaves as a "soft elastic membrane". In the linear anisotropic case, the local form of the transmission conditions is of Fourier Robin type. Let us also remark that this problem is a singular perturbation problem. For "mathematically oriented results", see e.g. [1,7,10,11,16,17].

The influence of a thin layer of high rigidity has been studied in different situations starting from [8] where the author identifies the weak limits for different orders of magnitude of the elastic coefficients, in the linear and isotropic case. In [1,14] and in [15] the authors study the limit problem for a surrounding layer, in the linear elasticity framework.

The aim of the present paper is to study the simplest "welding" situation for a three layers elastic system where a thin and strong material is inserted between two elastic materials. We examine two cases: in Section 3, the behavior of all the materials is linear elastic and anisotropic. In Section 4 we consider the usual non convex elastic potential, given by a Caratheodory function. The two cases are studied in the framework of the $\Gamma$-convergence. In the limit problem, the "material surface" behaves as a "stiffening" elastic membrane. In the linear anisotropic case, we write the local form of the transmission conditions which are of Ventcel type [18]. Let also explicitly remark that the "welding" problem is a regular perturbation problem.

\section{Problem statement}

In the three-dimensional Euclidean space $\mathbb{E}^{3}$ referred to the orthonormal frame $\left(0 ; \mathbf{e}_{1}, \mathbf{e}_{2}, \mathbf{e}_{3}\right)$, let $\Omega^{-}$ and $\Omega^{+}$be two disjoint domains with $C^{2}$ piecewise boundaries $\partial \Omega^{+}$and $\partial \Omega^{-}$. Let $S=\left\{\partial \Omega^{+} \cap \partial \Omega^{-}\right\}^{\circ}$ be the interior of the common part of the external surfaces which is assumed to have a positive $2 D$ measure and which is assumed to be projectable onto the plane $\left\{x_{3}=0\right\}$. We consider a multimaterial obtained by the insertion between the materials occupying $\Omega^{-}$and $\Omega^{+}$of a third material. For this, $\Omega^{+}$ (resp. $\Omega^{-}$) is moved in the $\mathbf{e}_{3}$ (resp. $-\mathbf{e}_{3}$ ) direction of an amount equal to the half-thickness $\frac{\varepsilon h}{2}$ of the third material, where $\varepsilon$ is a small dimensionless parameter and $h$ is a global characteristic length (for example the diameter of $\Omega$ ). Then, let $\Omega_{\varepsilon}^{ \pm}=\left\{\mathbf{x} \pm \frac{\varepsilon h}{2} \mathbf{e}_{3}, \mathbf{x} \in \Omega^{ \pm}\right\}, B_{\varepsilon}=\left\{\mathbf{x}+\varepsilon z \mathbf{e}_{3}, \frac{-h}{2}<z<\frac{h}{2}, \mathbf{x} \in S\right\}$, 

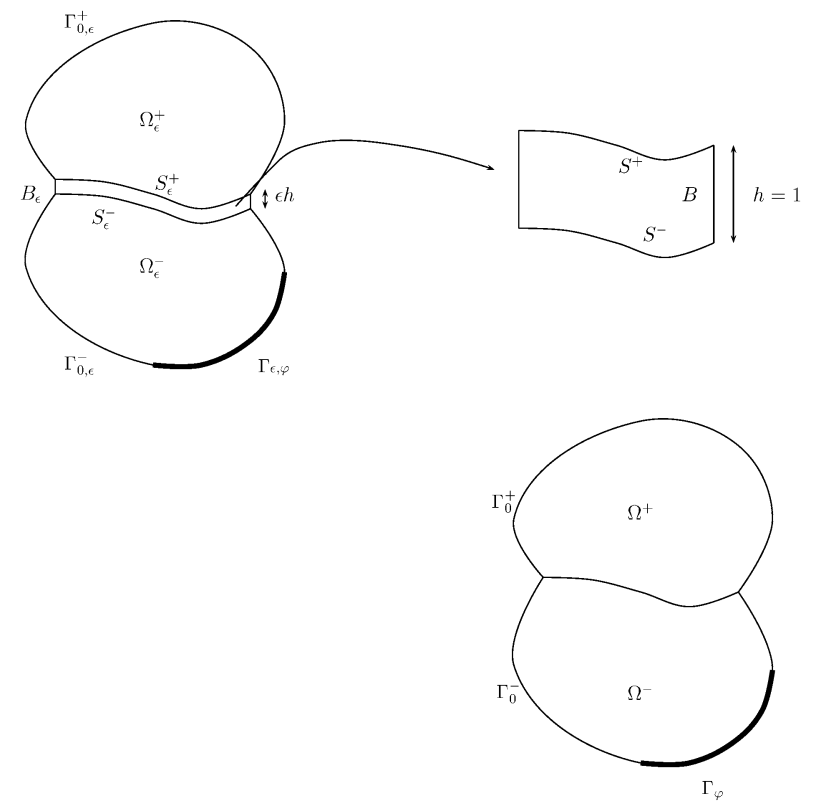

Fig. 1. Bonded assembly. Left: the physical configuration $\left(\Omega_{\varepsilon}\right)$. Right: the limit configuration $(\Omega)$. Below: the rescaled configuration.

$S_{\varepsilon}^{ \pm}=\left\{\mathbf{x} \pm \frac{\varepsilon h}{2} \mathbf{e}_{3}, \mathbf{x} \in S\right\}$ and $\Omega_{\varepsilon}=\Omega_{\varepsilon}^{+} \cup \Omega_{\varepsilon}^{-} \cup B_{\varepsilon} \cup S_{\varepsilon}^{+} \cup S_{\varepsilon}^{-}$be the physical reference configuration of the assembly (see Fig. 1 left). The structure is clamped on the parts $\Gamma_{0, \varepsilon}^{+} \subset \partial \Omega_{\varepsilon}^{+} \backslash S_{\varepsilon}^{+}$, resp. $\Gamma_{0, \varepsilon}^{-} \subset \partial \Omega_{\varepsilon}^{-} \backslash S_{\varepsilon}^{-}$, whose union is denoted by $\Gamma_{0, \varepsilon}$. The external boundary $\left\{\mathbf{x}+\frac{\varepsilon z}{2} \mathbf{e}_{3},-h<z<h, \mathbf{x} \in\right.$ $\partial S\}$ of $B_{\varepsilon}$ is traction-free. The complementary part $\Gamma_{\varepsilon, \varphi}$ of the boundary of $\Omega_{\varepsilon}$ is submitted to surface loads $\varphi_{\varepsilon}$. Obviously one can there consider other type of boundary conditions (e.g. a combination of some components of the stress vector and of the displacement). The structure is also submitted to applied body forces $\mathbf{f}_{\varepsilon}$.

The conventions of sum over repeated indexes and of comma representing derivative will be employed. Furthermore, latin indexes range in $\{1,2,3\}$ and greek indexes range in $\{1,2\}$.

The stored energy density, $\omega^{\varepsilon}\left(\mathbf{x}^{\varepsilon}, \nabla^{\varepsilon} \mathbf{v}\right)$, associated with a displacement field $\mathbf{v}: \Omega_{\varepsilon} \mapsto \mathbb{R}^{3}$, is defined by

$$
\omega^{\varepsilon}\left(\mathbf{x}^{\varepsilon}, \nabla^{\varepsilon} \mathbf{v}\right)= \begin{cases}f^{+}\left(\nabla^{\varepsilon} \mathbf{v}\right) & \text { in } \Omega_{\varepsilon}^{+}, \\ f^{-}\left(\nabla^{\varepsilon} \mathbf{v}\right) & \text { in } \Omega_{\varepsilon}^{-}, \\ \frac{1}{\varepsilon} g\left(\nabla^{\varepsilon} \mathbf{v}\right) & \text { in } B_{\varepsilon},\end{cases}
$$

where $\nabla^{\varepsilon} \mathbf{v}=\frac{\partial \mathbf{v}}{\partial \mathbf{x}^{\varepsilon}}$. Let us explicitely point out that the "adimensional" coefficient $\frac{1}{\varepsilon}$ in the stored energy of the thin layer $B_{\varepsilon}$ expresses the "welding" character of our model.

The stored strain energy associated with a displacement field $\mathbf{v}$ is given by the functional

$$
W_{\varepsilon}(\mathbf{v}):=\int_{\Omega_{\varepsilon}} \omega^{\varepsilon}\left(\mathbf{x}^{\varepsilon}, \nabla^{\varepsilon} \mathbf{v}\right) \mathrm{d} x .
$$


In the sequel $\mathbf{M}^{3 \times 3}$ denotes the set of $3 \times 3$ matrices. If $p \geqslant 1$, we say that a Borel function $w: \mathbf{M}^{3 \times 3} \rightarrow \mathbb{R}$ satisfies a $\left(C_{p}\right)$ condition if

$$
\left\{\begin{array}{l}
\exists \alpha, \beta, C \in \mathbb{R}^{+} \text {s.t. } \forall\left(\lambda, \lambda^{\prime}\right) \in \mathbf{M}^{3 \times 3}, \quad \alpha|\lambda|^{p} \leqslant w(\lambda) \leqslant \beta\left(1+|\lambda|^{p}\right), \\
\left|w(\lambda)-w\left(\lambda^{\prime}\right)\right| \leqslant C\left|\lambda-\lambda^{\prime}\right|\left(1+|\lambda|^{p-1}+\left|\lambda^{\prime}\right|^{p-1}\right) .
\end{array}\right.
$$

The bulk energy densities $f^{ \pm}$and $g$ of the materials occupying $\Omega_{\varepsilon}^{ \pm}$and $B_{\varepsilon}$ satisfy a $\left(C_{p}\right)$ condition with $p>1$ and to shorten notation we assume that $\left(C_{p}\right)$ is satisfied with the same constants $\alpha, \beta$ and $C$.

The equilibrium configuration of the structure is given by the displacement fields $\mathbf{u}_{\varepsilon}$, solution or, more generally, $\varepsilon$-approximate solution of the problem

$$
\inf \left\{J_{\varepsilon}(\mathbf{v}): \mathbf{v} \in W_{\Gamma_{0, \varepsilon}}^{1, p}\left(\Omega^{\varepsilon}, \mathbb{R}^{3}\right)\right\}
$$

where $J_{\varepsilon}(\mathbf{v}):=W_{\varepsilon}(\mathbf{v})-L_{\varepsilon}(\mathbf{v})$ is the total potential energy. As mentioned in the Introduction, our aim is to study the behavior of $\mathbf{u}^{\varepsilon}$ when $\varepsilon$ tends to zero and to identify the variational problem whose solution is a suitable limit of $\mathbf{u}^{\varepsilon}$. Our strategy consists in computing the $\Gamma$-limit of the sequence $\left(J_{\varepsilon}\right)_{\varepsilon>0}$. In order to make apparent the dependance on $\varepsilon$ of the problem, we define, following the approach of Ciarlet and Destuynder [9], an equivalent problem in the fixed domain $\Omega$ (see Fig. 1 right). For this purpose, we set $\pi^{\varepsilon}: \mathbf{x}=\left(x_{1}, x_{2}, x_{3}\right) \in \Omega \rightarrow \mathbf{x}^{\varepsilon}=\left(x_{1}^{\varepsilon}, x_{2}^{\varepsilon}, x_{3}^{\varepsilon}\right) \in \Omega_{\varepsilon}$ defined by

$$
\begin{cases}\pi^{\varepsilon}\left(x_{1}, x_{2}, x_{3}\right)=\left(x_{1}, x_{2}, x_{3}-\frac{h}{2}(1-\varepsilon)\right) \in \Omega_{\varepsilon}^{+}, & \text {for } \mathbf{x} \in \Omega_{\mathrm{tr}}^{+}, \\ \pi^{\varepsilon}\left(x_{1}, x_{2}, x_{3}\right)=\left(x_{1}, x_{2}, \varepsilon x_{3}\right) \in B_{\varepsilon}, & \text { for } \mathbf{x} \in B, \\ \pi^{\varepsilon}\left(x_{1}, x_{2}, x_{3}\right)=\left(x_{1}, x_{2}, x_{3}+\frac{h}{2}(1-\varepsilon)\right) \in \Omega_{\varepsilon}^{-}, & \text {for } \mathbf{x} \in \Omega_{\mathrm{tr}}^{-},\end{cases}
$$

with $\Omega_{\mathrm{tr}}^{ \pm}=\left\{\mathbf{x} \pm \frac{h}{2} \mathbf{e}_{3}, \mathbf{x} \in \Omega^{ \pm}\right\}, B=\left\{\mathbf{x}+\frac{z}{2} \mathbf{e}_{3},-h<z<h, \mathbf{x} \in S\right\}, S^{ \pm}=\left\{\mathbf{x} \pm \frac{h}{2} \mathbf{e}_{3}, \mathbf{x} \in S\right\}$. In order to simplify the notations, we identify $\Omega_{\mathrm{tr}}^{+}$with $\Omega^{+}$and $\Omega_{\mathrm{tr}}^{-}$with $\Omega^{-}$and denote $\Gamma_{0}$, resp $\Gamma_{\boldsymbol{\varphi}}$, the transformed of $\Gamma_{0, \varepsilon}$, resp. $\Gamma_{\varepsilon, \varphi}$. At last, we set $\Omega=\Omega^{+} \cup \Omega^{-} \cup B \cup S^{+} \cup S^{-}$. The displacement field, the external loads and the elastic properties of the bodies are then defined without rescaling by $\mathbf{v}(\varepsilon, \mathbf{x}):=\mathbf{v}^{\varepsilon}\left(\mathbf{x}^{\varepsilon}\right)=\mathbf{v}^{\varepsilon} o \pi^{\varepsilon}(\mathbf{x})$. Let us also recall the usual relations for any differentiable function $\phi^{\varepsilon}$ defined in $B_{\varepsilon}$ :

$$
\begin{aligned}
\frac{\partial}{\partial x_{\alpha}^{\varepsilon}}\left(\phi^{\varepsilon}\left(\mathbf{x}^{\varepsilon}\right)\right) & =\frac{\partial}{\partial x_{\alpha}}(\phi(\mathbf{x})), \\
\frac{\partial}{\partial x_{3}^{\varepsilon}}\left(\phi^{\varepsilon}\left(\mathbf{x}^{\varepsilon}\right)\right) & =\frac{1}{\varepsilon} \frac{\partial}{\partial x_{3}}(\phi(\mathbf{x}))
\end{aligned}
$$

and

$$
\int_{B_{\varepsilon}} \phi^{\varepsilon}\left(\mathbf{x}^{\varepsilon}\right) \mathrm{d} \mathbf{x}^{\varepsilon}=\varepsilon \int_{B} \phi^{\varepsilon} o \pi^{\varepsilon}(\mathbf{x}) \mathrm{d} \mathbf{x} \equiv \varepsilon \int_{B} \phi(\mathbf{x}) \mathrm{d} \mathbf{x} .
$$


With these assumptions the functional $J_{\varepsilon}(\mathbf{v})$ defined on $W_{\Gamma_{0, \varepsilon}}^{1, p}\left(\Omega^{\varepsilon}, \mathbb{R}^{3}\right)$ is transformed into the following functional $J(\varepsilon, \mathbf{v})$ defined on $W_{\Gamma_{0}}^{1, p}\left(\Omega, \mathbb{R}^{3}\right)$ by

$$
J(\varepsilon, \mathbf{v})=\int_{\Omega^{-}} f^{-}(\nabla \mathbf{v}) \mathrm{d} x+\int_{\Omega^{+}} f^{+}(\nabla \mathbf{v}) \mathrm{d} x+\int_{B} g\left(\hat{\nabla} \mathbf{v} \mid \frac{1}{\varepsilon} \frac{\partial \mathbf{v}}{\partial x_{3}}\right) \mathrm{d} x-L(\mathbf{v})-\varepsilon L^{s}(\mathbf{v})
$$

where $\hat{\nabla} \mathbf{v}=\left(\frac{\partial v_{i}}{\partial x_{\alpha}}\right)_{i=1,2,3, \alpha=1,2}$.

\section{A linear anisotropic model}

In the linear hyperelastic case, the bulk energy density can be taken as a function of the linearized strain tensor defined by

$$
\mathbf{e}\left(\mathbf{u}^{\varepsilon}\right)=\frac{1}{2}\left(\nabla \mathbf{u}^{\varepsilon}+\left(\nabla \mathbf{u}^{\varepsilon}\right)^{\top}\right)
$$

and the stress tensor $\boldsymbol{\sigma}^{\varepsilon}\left(\mathbf{x}^{\varepsilon}\right)$ satisfies the constitutive relation

$$
\boldsymbol{\sigma}^{\varepsilon}\left(\mathbf{x}^{\varepsilon}\right)=\frac{\partial W^{\varepsilon}}{\partial \mathbf{e}}\left(\mathbf{x}^{\varepsilon}, \mathbf{e}\left(\mathbf{u}^{\varepsilon}\right)\right)
$$

where $\omega^{\varepsilon}$ denotes the stored energy density defined in our situation by

$$
\omega^{\varepsilon}\left(\mathbf{x}^{\varepsilon}, \mathbf{e}\right)=\frac{1}{2} \mathbf{A}\left(\mathbf{x}^{\varepsilon}\right) \mathbf{e}: \mathbf{e}= \begin{cases}\frac{1}{2} \mathbf{A}^{+} \mathbf{e}: \mathbf{e} & \text { in } \Omega_{\varepsilon}^{+}, \\ \frac{1}{2} \mathbf{A}^{-} \mathbf{e}: \mathbf{e} & \text { in } \Omega_{\varepsilon}^{-}, \\ \frac{1}{2 \varepsilon} \mathbf{A}^{s} \mathbf{e}: \mathbf{e} & \text { in } B_{\varepsilon},\end{cases}
$$

and $\mathbf{A}\left(\mathbf{x}^{\varepsilon}\right)$ is the hyperelastic fourth-order tensor satisfying the usual symmetry, boundedness and positivity assumptions. Under these assumptions the $\left(C_{2}\right)$ condition is satisfied and one can prove that the minimization problem (2) has a unique solution $\mathbf{u}^{\varepsilon}$. Here the potential energy is

$$
J_{\varepsilon}(\mathbf{v}):=\frac{1}{2} a_{\varepsilon}^{+}(\mathbf{v}, \mathbf{v})+\frac{1}{2} a_{\varepsilon}^{-}(\mathbf{v}, \mathbf{v})+\frac{1}{2 \varepsilon} a_{\varepsilon}^{s}(\mathbf{v}, \mathbf{v})-L_{\varepsilon}(\mathbf{v}),
$$

where

$$
\begin{aligned}
& a_{\varepsilon}^{ \pm}(\mathbf{v}, \mathbf{v}):=\int_{\Omega_{\varepsilon}^{ \pm}} \mathbf{A}^{ \pm} \mathbf{e}(\mathbf{v}): \mathbf{e}(\mathbf{v}) \mathrm{d} x, \\
& a_{\varepsilon}^{s}(\mathbf{v}, \mathbf{v}):=\int_{B_{\varepsilon}} \mathbf{A}^{s} \mathbf{e}(\mathbf{v}): \mathbf{e}(\mathbf{v}) \mathrm{d} x, \\
& L_{\varepsilon}(\mathbf{v}):=\int_{\Omega_{\varepsilon}} \mathbf{f}_{\varepsilon} \mathbf{v} \mathrm{d} x+\int_{\Gamma_{\varepsilon, \varphi}} \boldsymbol{\varphi}_{\varepsilon} \mathbf{v} \mathrm{d} \Gamma .
\end{aligned}
$$


Using the transformation (3), $J_{\varepsilon}(\mathbf{v})$ defined on $W_{\Gamma_{0, \varepsilon}}^{1,2}\left(\Omega^{\varepsilon}, \mathbb{R}^{3}\right)$ is converted into the functional $J(\varepsilon, \mathbf{v})$ defined on $W_{\Gamma_{0}}^{1,2}\left(\Omega, \mathbb{R}^{3}\right)$, see (4). More explicitly

$$
J(\varepsilon, \mathbf{v})=\frac{1}{2} a^{+}(\mathbf{v}, \mathbf{v})+\frac{1}{2} a^{-}(\mathbf{v}, \mathbf{v})+\frac{1}{2} a_{\alpha \beta}^{s}(\mathbf{v}, \mathbf{v})-L(\mathbf{v})+\frac{1}{2 \varepsilon^{2}} a_{33}^{s}(\mathbf{v}, \mathbf{v})+\frac{1}{2 \varepsilon} a_{3 \alpha}^{s}(\mathbf{v}, \mathbf{v})-\varepsilon L^{s}(\mathbf{v}),
$$

where

$$
\begin{aligned}
& a^{ \pm}(\mathbf{v}, \mathbf{v}):=\int_{\Omega^{ \pm}} \mathbf{A}^{ \pm} \mathbf{e}(\mathbf{v}): \mathbf{e}(\mathbf{v}) \mathrm{d} x, \\
& a_{\alpha \beta}^{s}(\mathbf{v}, \mathbf{v}):=\int_{B} A_{i \alpha h \beta}^{s} v_{i, \alpha} v_{h, \beta} \mathrm{d} x, \\
& a_{3 \alpha}^{s}(\mathbf{v}, \mathbf{v}):=2 \int_{B} A_{i 3 h \alpha}^{s} v_{i, 3} v_{h, \alpha} \mathrm{d} x, \\
& a_{33}^{s}(\mathbf{v}, \mathbf{v}):=\int_{B} A_{i 3 h 3}^{s} v_{i, 3} v_{h, 3} \mathrm{~d} x, \\
& L(\mathbf{v}):=\int_{\Omega^{+} \cup \Omega^{-}} \mathbf{f v} \mathrm{d} x+\int_{\Gamma_{\varphi}} \varphi \mathbf{v} \mathrm{d} \Gamma, \\
& L^{s}(\mathbf{v}):=\int_{B} \mathbf{f v} \mathrm{d} x .
\end{aligned}
$$

The problem (2) becomes:

Find $\mathbf{u}(\varepsilon) \in W_{\Gamma_{0}}^{1,2}\left(\Omega, \mathbb{R}^{3}\right)$ such that

$$
J(\varepsilon, \mathbf{u}(\varepsilon)) \leqslant J(\varepsilon, \mathbf{v}), \quad \forall \mathbf{v} \in W_{\Gamma_{0}}^{1,2}\left(\Omega, \mathbb{R}^{3}\right) .
$$

Obviously, this problem still has a unique solution satisfying

$$
\begin{aligned}
& a^{+}(\mathbf{u}(\varepsilon), \mathbf{u}(\varepsilon))+a^{-}(\mathbf{u}(\varepsilon), \mathbf{u}(\varepsilon))+a_{\alpha \beta}^{s}(\mathbf{u}(\varepsilon), \mathbf{u}(\varepsilon))+\frac{1}{\varepsilon} a_{3 \alpha}^{s}(\mathbf{u}(\varepsilon), \mathbf{u}(\varepsilon))+\frac{1}{\varepsilon^{2}} a_{33}^{s}(\mathbf{u}(\varepsilon), \mathbf{u}(\varepsilon)) \\
& \quad=L(\mathbf{u}(\varepsilon))+\varepsilon L^{s}(\mathbf{u}(\varepsilon)) .
\end{aligned}
$$

\subsection{A priori estimates}

From (6) and thanks to the coercivity and boundedness assumptions, we obtain the following a priori estimates, and from now on we use the same notation $C$ to denote various constants.

Proposition 1. The solution $\mathbf{u}(\varepsilon)$ of problem (5) fulfills the following estimates:

$$
\begin{aligned}
& \|\mathbf{u}(\varepsilon)\|_{1, \Omega^{ \pm}}^{2} \leqslant C, \\
& \|\mathbf{u}(\varepsilon)\|_{0, B}^{2} \leqslant C, \\
& \frac{1}{\varepsilon^{2}}\left\|u(\varepsilon)_{i, 3}\right\|_{0, B}^{2} \leqslant C, \\
& \left\|u(\varepsilon)_{i, \alpha}\right\|_{0, B}^{2} \leqslant C .
\end{aligned}
$$


Proof. The proposition is a straightforward consequence of the coercivity of the bilinear form, the Korn inequality and of assumptions on the surface loading.

Corollary 1. There exist $\mathbf{u} \in W_{\Gamma_{0}}^{1,2}\left(\Omega, \mathbb{R}^{3}\right), \mathbf{w} \in L^{2}\left(B, \mathbb{R}^{3}\right)$ and a subsequence of $(\mathbf{u}(\varepsilon))_{\varepsilon>0}$ not relabeled such that:

(i) $\mathbf{u}(\varepsilon) \rightarrow \mathbf{u}$ weakly in $W_{\Gamma_{0}}^{1,2}\left(\Omega, \mathbb{R}^{3}\right)$ and strongly in $L^{2}\left(\Omega, \mathbb{R}^{3}\right)$;

(ii) $u(\varepsilon)_{i, 3} \rightarrow 0$ strongly in $L^{2}(B)$, and so $u_{i, 3}=0$ in $B$;

(iii) $\frac{1}{\varepsilon} u(\varepsilon)_{i, 3} \rightarrow w_{i}$ weakly in $L^{2}(B)$.

We have now to identify the problem satisfied by $\mathbf{u}$. This will be obtained in the framework of $\Gamma$-convergence. Moreover, since the previous corollary implies that the limit problem will not be a singular perturbation of the original one, we will also prove the convergence of $\mathbf{u}(\varepsilon)$ in $W_{\Gamma_{0}}^{1,2}\left(\Omega, \mathbb{R}^{3}\right)$ for the strong topology.

\section{2. $\Gamma$-convergence}

Let us introduce the functionals $\mathcal{F}_{\varepsilon}: L^{2}\left(\Omega, \mathbb{R}^{3}\right) \rightarrow \mathbb{R} \cup\{+\infty\}$ and $\mathcal{F}: L^{2}\left(\Omega, \mathbb{R}^{3}\right) \rightarrow \mathbb{R} \cup\{+\infty\}$ given by

$$
\mathcal{F}_{\varepsilon}(\mathbf{v}):= \begin{cases}J(\varepsilon, \mathbf{v}) & \text { if } \mathbf{v} \in W_{\Gamma_{0}}^{1,2}\left(\Omega, \mathbb{R}^{3}\right) \\ +\infty & \text { otherwise }\end{cases}
$$

and

$$
\mathcal{F}(\mathbf{v}):= \begin{cases}\frac{1}{2} a^{+}(\mathbf{v}, \mathbf{v})+\frac{1}{2} a^{-}(\mathbf{v}, \mathbf{v})-L(\mathbf{v})+\frac{1}{2} J_{0}^{s}(\mathbf{v}) & \text { if } \mathbf{v} \in V_{B}, \\ +\infty & \text { otherwise }\end{cases}
$$

where

$$
V_{B}:=\left\{\mathbf{v} \in W_{\Gamma_{0}}^{1,2}\left(\Omega, \mathbb{R}^{3}\right) ; v_{i, 3}=0 \text { in } B\right\}
$$

and

$$
J_{0}^{s}(\mathbf{v}):=a_{\alpha \beta}^{s}(\mathbf{v}, \mathbf{v})+Q^{s}(\mathbf{v})
$$

with

$$
Q^{s}(\mathbf{v}):=\inf _{\boldsymbol{\zeta} \in L^{2}\left(B, \mathbb{R}^{3}\right)}\left(\int_{B} A_{i 3 h 3}^{s} \zeta_{i} \zeta_{h} \mathrm{~d} x+2 \int_{B} A_{i 3 h \alpha}^{s} \zeta_{i} v_{h, \alpha} \mathrm{d} x\right) .
$$

Proposition 2. The functional $J_{0}^{s}$ is quadratic and satisfies for all $\mathbf{v} \in V_{B}$

$$
0 \leqslant J_{0}^{s}(\mathbf{v}) \leqslant a_{\alpha \beta}^{s}(\mathbf{v}, \mathbf{v}) .
$$

Consequently $\mathbf{v} \mapsto J_{0}^{s}(\mathbf{v})$ is a convex functional. 
Proof. For all $\mathbf{v} \in V_{B}$ one has $Q^{s}(\mathbf{v}) \leqslant 0$, hence the second inequality of (8). In order to prove that $J_{0}^{s}(\mathbf{v})$ is quadratic and the first inequality of (8) we find at first an explicit form for $Q^{s}$. Since the tensor $\mathbf{M}$ whose components are $(\mathbf{M})_{i j}:=A_{i 3 j 3}^{s}$ is coercive, for every $\mathbf{v} \in V_{B}$ the infimum in (7) is attained for $\zeta=\mathbf{w}(\mathbf{v})$, where

$$
w_{i}(\mathbf{v})=-\left(M^{-1}\right)_{i j} A_{j 3 h \alpha}^{s} v_{h, \alpha}
$$

Let us remark that $\mathbf{w}(\mathbf{v})$ does not depend on $x_{3}$, hence can be identified with an element of $L^{2}\left(S, \mathbb{R}^{3}\right)$. Moreover,

$$
Q^{s}(\mathbf{v})=-\int_{B}\left(M^{-1}\right)_{i j} A_{i 3 h \alpha}^{s} v_{h, \alpha} A_{j 3 k \beta}^{s} v_{k, \beta} \mathrm{d} x
$$

so that $\mathbf{v} \mapsto J_{0}^{s}(\mathbf{v})$ is a quadratic form. The first inequality in (8) is a straightforward consequence of the properties of the tensor $\mathbf{A}^{s}$. Let $\mathbf{M}^{3 \times 2}$ denote the set of $3 \times 2$ matrices. For $\boldsymbol{\tau}=\left(\tau_{i j}\right) \in \mathbf{M}^{3 \times 3}$, we write $\boldsymbol{\tau}=\left(\tau_{1}\left|\tau_{2}\right| \tau_{3}\right)=\left(\hat{\boldsymbol{\tau}} \mid \tau_{3}\right)$, where $\hat{\boldsymbol{\tau}} \in \mathbf{M}^{3 \times 2}$ and we denote by $\boldsymbol{\tau}^{S}=\frac{1}{2}\left(\left(\tau_{i j}+\tau_{j i}\right)\right)$ its symmetrized part. Symmetry and coercivity properties of $\mathbf{A}^{s}$ imply that there exists $\kappa^{s}>0$ such that for all matrix $\tau \in \mathbf{M}^{3 \times 3}$ :

$$
\mathbf{A}^{s} \boldsymbol{\tau}: \boldsymbol{\tau}=A_{i j h k}^{s} \tau_{i j} \tau_{h k}=\mathbf{A}^{s} \boldsymbol{\tau}^{S}: \boldsymbol{\tau}^{S} \geqslant \kappa^{s} \boldsymbol{\tau}^{S}: \boldsymbol{\tau}^{S} \geqslant 0
$$

It follows that

$$
\begin{aligned}
0 & \leqslant \inf _{\tau_{3} \in \mathbb{R}^{3}} \mathbf{A}^{s} \boldsymbol{\tau}: \boldsymbol{\tau}=\inf _{\tau_{3} \in \mathbb{R}^{3}} A_{i j h k}^{s} \tau_{i j} \tau_{h k} \\
& =A_{i \alpha h \beta}^{s} \tau_{i \alpha} \tau_{h \beta}+\inf _{\tau_{3} \in \mathbb{R}^{3}}\left(A_{i 3 h 3}^{s} \tau_{i 3} \tau_{h 3}+2 A_{i 3 h \beta}^{s} \tau_{i 3} \tau_{h \beta}\right) \\
& =A_{i \alpha h \beta}^{s} \tau_{i \alpha} \tau_{h \beta}-\left(M^{-1}\right)_{i j} A_{i 3 h \alpha}^{s} \tau_{h \alpha} A_{j 3 k \beta}^{s} \tau_{k \beta} .
\end{aligned}
$$

For $\hat{\boldsymbol{\tau}} \in \mathbf{M}^{3 \times 2}$ define the function $g_{0}$ by

$$
g_{0}(\hat{\tau}):=A_{i \alpha h \beta}^{s} \tau_{i \alpha} \tau_{h \beta}-\left(M^{-1}\right)_{i j} A_{i 3 h \alpha}^{s} \tau_{h \alpha} A_{j 3 k \beta}^{s} \tau_{k \beta} .
$$

From (11) it follows that $g_{0}$ is positive (and quadratic), hence

$$
J_{0}^{s}(\mathbf{v})=\int_{B} g_{0}(\hat{\nabla} \mathbf{v}) \mathrm{d} x
$$

satisfies the first inequality of (8).

Theorem 1. Let us equip the space $L^{2}\left(\Omega, \mathbb{R}^{3}\right)$ with the strong topology. Under the previous assumptions, the sequence $\left(\mathcal{F}_{\varepsilon}\right)_{\varepsilon>0} \Gamma$-converges to $\mathcal{F}$.

The proof consists in establishing Propositions 3 and 4. 
Proposition 3. For all $\mathbf{v} \in L^{2}\left(\Omega, \mathbb{R}^{3}\right)$ and all sequence $\left(\mathbf{v}_{\varepsilon}\right)_{\varepsilon>0}$ strongly converging to $\mathbf{v}$ in $L^{2}\left(\Omega, \mathbb{R}^{3}\right)$ the following inequality holds:

$$
\mathcal{F}(\mathbf{v}) \leqslant \liminf _{\varepsilon \rightarrow 0} \mathcal{F}_{\varepsilon}\left(\mathbf{v}_{\varepsilon}\right)
$$

Proof. Clearly one may assume that $\liminf _{\varepsilon \rightarrow 0} \mathcal{F}_{\varepsilon}\left(\mathbf{v}_{\varepsilon}\right)<+\infty$. Therefore $\left(\mathbf{v}_{\varepsilon}\right)_{\varepsilon>0}$ fulfills the a priori estimates of Proposition 1. Using Corollary 1 we deduce that $v_{i, 3}=0$ in $B$ and that $\left(\frac{1}{\varepsilon} \mathbf{v}_{\varepsilon}\right)_{, 3}$ weakly converges to some function denoted $\boldsymbol{z}$ in $L^{2}\left(B, \mathbb{R}^{3}\right)$. We can write $J\left(\varepsilon, \mathbf{v}_{\varepsilon}\right)$ as follows

$$
J\left(\varepsilon, \mathbf{v}_{\varepsilon}\right)=\hat{J}\left(\mathbf{v}_{\varepsilon},\left(\frac{1}{\varepsilon} \mathbf{v}_{\varepsilon}\right)_{, 3}\right)-L\left(\mathbf{v}_{\varepsilon}\right)-\varepsilon L^{s}\left(\mathbf{v}_{\varepsilon}\right)
$$

where

$$
\hat{J}(\mathbf{v}, \zeta):=\frac{1}{2} a^{+}(\mathbf{v}, \mathbf{v})+\frac{1}{2} a^{-}(\mathbf{v}, \mathbf{v})+\frac{1}{2} a_{\alpha \beta}^{s}(\mathbf{v}, \mathbf{v})+\frac{1}{2} \int_{B} A_{i 3 j 3}^{s} \zeta_{i} \zeta_{j} \mathrm{~d} x+\int_{B} A_{i 3 h \alpha}^{s} \zeta_{i} v_{h, \alpha} \mathrm{d} x .
$$

By lower semi-continuity we obtain $\liminf _{\varepsilon \rightarrow 0} \mathcal{F}_{\varepsilon}\left(\mathbf{v}_{\varepsilon}\right) \geqslant \hat{J}(\mathbf{v}, \boldsymbol{z})-L(\mathbf{v}) \geqslant \mathcal{F}(\mathbf{v})$.

Proposition 4. For all $\mathbf{v} \in L^{2}\left(\Omega, \mathbb{R}^{3}\right)$ there exists a sequence $\left(\mathbf{v}_{\varepsilon}\right)_{\varepsilon>0}$ strongly converging to $\mathbf{v}$ in $L^{2}\left(\Omega, \mathbb{R}^{3}\right)$ such that

$$
\lim _{\varepsilon \rightarrow 0} \mathcal{F}_{\varepsilon}\left(\mathbf{v}_{\varepsilon}\right)=\mathcal{F}(\mathbf{v})
$$

Proof. Let $\mathbf{v}$ be a fixed element in $L^{2}\left(\Omega, \mathbb{R}^{3}\right)$. One may assume $\mathcal{F}(v)<+\infty$ otherwise there is nothing to prove. Hence we can assume that $\mathbf{v} \in V_{B}$. Let $\left(\boldsymbol{\omega}^{\eta}\right)_{\eta>0}$ be a sequence in $\mathcal{D}\left(S, \mathbb{R}^{3}\right)$ strongly converging to $\mathbf{w}(\mathbf{v})$, (see (9)), in $L^{2}\left(S, \mathbb{R}^{3}\right)$. Let $\left(\phi^{\eta}\right)_{\eta>0} \in \mathcal{D}\left(\Omega, \mathbb{R}^{3}\right)$ such that $\phi^{\eta}(x)=x_{3} \boldsymbol{\omega}^{\eta}$ in $B$ and define $\mathbf{v}_{\varepsilon}^{\eta}=\mathbf{v}+\varepsilon \boldsymbol{\phi}^{\eta}$. Then $\mathbf{v}_{\varepsilon}^{\eta} \in W_{\Gamma_{0}}^{1,2}\left(\Omega, \mathbb{R}^{3}\right)$ and

$$
\begin{aligned}
& a^{ \pm}\left(\mathbf{v}_{\varepsilon}^{\eta}, \mathbf{v}_{\varepsilon}^{\eta}\right)=a^{ \pm}(\mathbf{v}, \mathbf{v})+2 \varepsilon a^{ \pm}\left(\phi^{\eta}, \mathbf{v}\right)+\varepsilon^{2} a^{ \pm}\left(\phi^{\eta}, \phi^{\eta}\right), \\
& a_{\alpha \beta}^{s}\left(\mathbf{v}_{\varepsilon}^{\eta}, \mathbf{v}_{\varepsilon}^{\eta}\right)=a_{\alpha \beta}^{s}(\mathbf{v}, \mathbf{v})+2 \varepsilon a_{\alpha \beta}^{s}\left(\phi^{\eta}, \mathbf{v}\right)+\varepsilon^{2} a_{\alpha \beta}^{s}\left(\phi^{\eta}, \phi^{\eta}\right), \\
& a_{3 \alpha}^{s}\left(\mathbf{v}_{\varepsilon}^{\eta}, \mathbf{v}_{\varepsilon}^{\eta}\right)=\varepsilon a_{3 \alpha}^{s}\left(\phi^{\eta}, \mathbf{v}\right)+\varepsilon^{2} a_{3 \alpha}^{s}\left(\phi^{\eta}, \phi^{\eta}\right), \\
& a_{33}^{s}\left(\mathbf{v}_{\varepsilon}^{\eta}, \mathbf{v}_{\varepsilon}^{\eta}\right)=\varepsilon^{2} a_{33}^{s}\left(\phi^{\eta}, \phi^{\eta}\right) .
\end{aligned}
$$

We deduce

$$
\begin{aligned}
\lim _{\varepsilon \rightarrow 0} \mathcal{F}_{\varepsilon}\left(\mathbf{v}_{\varepsilon}^{\eta}\right)= & \frac{1}{2} a^{+}(\mathbf{v}, \mathbf{v})+\frac{1}{2} a^{-}(\mathbf{v}, \mathbf{v})-L(\mathbf{v}) \\
& +\frac{1}{2}\left\{a_{\alpha \beta}^{s}(\mathbf{v}, \mathbf{v})+2 \int_{B} A_{i 3 h \alpha}^{s} \omega_{i}^{\eta} v_{h, \alpha} \mathrm{d} x+\int_{B} A_{i 3 h 3}^{s} \omega_{i}^{\eta} \omega_{h}^{\eta} \mathrm{d} x\right\} .
\end{aligned}
$$


Since the sequence $\left(\boldsymbol{\omega}^{\eta}\right)_{\eta>0}$ strongly converges to $\mathbf{w}(\mathbf{v})$ in $L^{2}\left(S, \mathbb{R}^{3}\right)$ it follows

$$
\begin{aligned}
\lim _{\eta \rightarrow 0} \lim _{\varepsilon \rightarrow 0} \mathcal{F}_{\varepsilon}\left(\mathbf{v}_{\varepsilon}^{\eta}\right)= & \frac{1}{2} a^{+}(\mathbf{v}, \mathbf{v})+\frac{1}{2} a^{-}(\mathbf{v}, \mathbf{v})-L(\mathbf{v}) \\
& +\frac{1}{2}\left\{a_{\alpha \beta}^{s}(\mathbf{v}, \mathbf{v})+2 \int_{B} A_{i 3 h \alpha}^{s} w_{i}(\mathbf{v}) v_{h, \alpha} \mathrm{d} x+\int_{B} A_{i 3 h 3}^{s} w_{i}(\mathbf{v}) w_{h}(\mathbf{v}) \mathrm{d} x\right\}=\mathcal{F}(\mathbf{v}) .
\end{aligned}
$$

Then, using a standard diagonalization argument, there exists a map $\varepsilon \mapsto \eta(\varepsilon)$ satisfying $\eta(\varepsilon) \rightarrow 0$ whenever $\varepsilon \rightarrow 0$ for which one has

$$
\lim _{\varepsilon \rightarrow 0} \mathcal{F}_{\varepsilon}\left(\mathbf{v}_{\varepsilon}^{\eta(\varepsilon)}\right)=\mathcal{F}(\mathbf{v})
$$

In order to complete the proof one only has to remark that the sequence $\left(\mathbf{v}_{\varepsilon}^{\eta(\varepsilon)}\right)_{\varepsilon>0}$ strongly converges to $\mathbf{v}$ in $L^{2}\left(\Omega, \mathbb{R}^{3}\right)$.

\subsection{Strong convergence}

Theorem 2. Under the previous assumptions, there exists a subsequence of $(\mathbf{u}(\varepsilon))_{\varepsilon>0}$ not relabeled such that $\mathbf{u}(\varepsilon)-\mathbf{u} \rightarrow 0$ strongly in $W_{\Gamma_{0}}^{1,2}\left(\Omega, \mathbb{R}^{3}\right)$.

Proof. Let $\left(\boldsymbol{\omega}^{\eta}\right)_{\eta>0}$ be a sequence in $\mathcal{D}\left(S, \mathbb{R}^{3}\right)$ strongly converging to $\mathbf{w}(\mathbf{u})$, see (9), in $L^{2}\left(S, \mathbb{R}^{3}\right)$ and $\left(\phi^{\eta}\right)_{\eta>0} \in \mathcal{D}\left(\Omega, \mathbb{R}^{3}\right)$ such that $\phi^{\eta}(\mathbf{x})=x_{3} \boldsymbol{\omega}^{\eta}(\hat{\mathbf{x}})$ in $B$. Then $\mathbf{u}(\varepsilon)-\mathbf{u}-\varepsilon \boldsymbol{\phi}^{\eta} \in W_{\Gamma_{0}}^{1,2}\left(\Omega, \mathbb{R}^{3}\right)$. Setting

$$
\begin{aligned}
& a\left(\mathbf{u}(\varepsilon)-\mathbf{u}-\varepsilon \boldsymbol{\phi}^{\eta}, \mathbf{u}(\varepsilon)-\mathbf{u}-\varepsilon \boldsymbol{\phi}^{\eta}\right) \\
& \quad:=a^{ \pm}\left(\mathbf{u}(\varepsilon)-\mathbf{u}-\varepsilon \boldsymbol{\phi}^{\eta}, \mathbf{u}(\varepsilon)-\mathbf{u}-\varepsilon \boldsymbol{\phi}^{\eta}\right)+a_{\alpha \beta}^{s}\left(\mathbf{u}(\varepsilon)-\mathbf{u}-\varepsilon \boldsymbol{\phi}^{\eta}, \mathbf{u}(\varepsilon)-\mathbf{u}-\varepsilon \boldsymbol{\phi}^{\eta}\right) \\
& \quad+\frac{1}{\varepsilon} a_{3 \alpha}^{s}\left(\mathbf{u}(\varepsilon)-\mathbf{u}-\varepsilon \boldsymbol{\phi}^{\eta}, \mathbf{u}(\varepsilon)-\mathbf{u}-\varepsilon \boldsymbol{\phi}^{\eta}\right)+\frac{1}{\varepsilon^{2}} a_{33}^{s}\left(\mathbf{u}(\varepsilon)-\mathbf{u}-\varepsilon \boldsymbol{\phi}^{\eta}, \mathbf{u}(\varepsilon)-\mathbf{u}-\varepsilon \boldsymbol{\phi}^{\eta}\right)
\end{aligned}
$$

a careful elementary computation gives

$$
\begin{aligned}
& \lim _{\varepsilon \rightarrow 0}\left\{a\left(\mathbf{u}(\varepsilon)-\mathbf{u}-\varepsilon \phi^{\eta}, \mathbf{u}(\varepsilon)-\mathbf{u}-\varepsilon \phi^{\eta}\right)\right\} \\
& \quad=-h \int_{S} A_{i 3 k 3}^{s} \omega_{i}^{\eta}\left(w_{k}-\omega_{k}^{\eta}\right) \mathrm{d} \hat{x}-h \int_{S} A_{k \alpha i 3}^{s} u_{k, \alpha}\left(w_{i}-\omega_{i}^{\eta}\right) \mathrm{d} \hat{x} .
\end{aligned}
$$

Since the sequence $\left(\boldsymbol{\omega}^{\eta}\right)_{\eta>0}$ strongly converges to $\mathbf{w}(\mathbf{u})$ in $L^{2}\left(S, \mathbb{R}^{3}\right)$ it follows

$$
\lim _{\eta \rightarrow 0}\left\{\int_{S} A_{i 3 k 3}^{s} \omega_{i}^{\eta}\left(w_{k}-\omega_{k}^{\eta}\right) \mathrm{d} \hat{x}+\int_{S} A_{k \alpha i 3}^{s} u_{k, \alpha}\left(w_{i}-\omega_{i}^{\eta}\right) \mathrm{d} \hat{x}=0\right\} .
$$

Using a standard diagonalization argument, there exists a map $\varepsilon \mapsto \eta(\varepsilon)$ satisfying $\eta(\varepsilon) \rightarrow 0$ whenever $\varepsilon \rightarrow 0$ for which one has

$$
\lim _{\varepsilon \rightarrow 0}\left\{a\left(\mathbf{u}(\varepsilon)-\mathbf{u}-\varepsilon \boldsymbol{\phi}^{\eta(\varepsilon)}, \mathbf{u}(\varepsilon)-\mathbf{u}-\varepsilon \boldsymbol{\phi}^{\eta(\varepsilon)}\right)\right\}=0 .
$$

The statement follows from the coercivity of the bilinear forms. 
Let us explicitly remark that indeed we have also given a characterization of the weak limit $\mathbf{w}$ established in Corollary 1 as $\mathbf{w}=\mathbf{w}(\mathbf{u})$ since

$$
\left(\frac{1}{\varepsilon} u(\varepsilon)_{i, 3}-w_{i}\right) \rightarrow 0 \quad \text { strongly in } L^{2}(B) .
$$

\subsection{The limit problem}

Classical results on $\Gamma$-convergence of the energies imply the convergence of minimum points and minimum values. Consequently, the limit problem is given by

Find $\mathbf{u} \in V_{B}$ so that

$$
\mathcal{F}(\mathbf{u}) \leqslant \mathcal{F}(\mathbf{v}), \quad \forall \mathbf{v} \in V_{B} .
$$

At first let us remark that this problem possesses a unique solution so that all the sequence $\mathbf{u}(\varepsilon)$ converges to $\mathbf{u}$. Under formulation (16) the limit problem is defined over $\Omega$, i.e., only on the adherents. This is one of the advantages of the simplified model: the intermediate layer disappears from a geometrical point of view, and it is described only by means of the surface energy. The differential formulation of (16) is that of a classical elastostatic problem with particular transmission conditions on $S$. Let us recall the classical decomposition: $\boldsymbol{\sigma} \mathbf{n}=\sigma_{N} \mathbf{n}+\boldsymbol{\sigma}_{\mathbf{T}}, \mathbf{n}$ being the normal vector to $S$. Then the transmission conditions on $S$ are

$$
\begin{cases}{[\mathbf{u}]=\mathbf{u}^{+}-\mathbf{u}^{-}=0} & \text { on } S \\ {[\boldsymbol{\sigma} \mathbf{n}]=\boldsymbol{\sigma}^{+} \mathbf{n}-\boldsymbol{\sigma}^{-} \mathbf{n}=h \operatorname{div}_{2} \widehat{\boldsymbol{\sigma}}} & \text { on } S\end{cases}
$$

where

$$
\widehat{\sigma}_{h \beta}=A_{i \alpha h \beta}^{s} u_{i, \alpha}-A_{j 3 h \beta}^{s}\left(M^{-1}\right)_{i j} A_{i 3 k \alpha}^{s} u_{k, \alpha}
$$

and

$$
\left(\operatorname{div}_{2} \widehat{\boldsymbol{\sigma}}\right)_{i}=\widehat{\sigma}_{i \beta, \beta}
$$

These transmission conditions are called of Ventcel type.

\section{A nonlinear model}

We make the assumption that the strain of the soft material can be high and that the thin structure $B_{\varepsilon}$ is occupied by a material which undergoes reversible solid-solid phase transformation as for instance crystalline solids. In this context, the densities $f$ and $g$ are not convex and may entail a multi-well structure. From this point of view the model suggested here is the macroscopic version of a model proposed in [6]. It is worth pointing out that the assumed growth condition violates the mechanical principle which asserts that it needs infinite amount of energy to squeeze a small piece of material down to a point. We also do not take into account preservation of orientation and injectivity conditions on the deformation fields so that the model presented in this section is a first attempt to account large purely 
elastic deformation. We hope to deal with this much more complex situation in a future work (for some results where these constraints are taken into account, we refer the reader to $[2,13])$.

As in Section 3, the change of scale (3) leads to consider the following functional:

$$
\begin{aligned}
& \mathcal{F}_{\varepsilon}: L^{p}\left(\Omega, \mathbb{R}^{3}\right) \rightarrow \mathbb{R} \cup\{+\infty\} \\
& \mathcal{F}_{\varepsilon}(\mathbf{v}):= \begin{cases}\int_{\Omega^{-}} f^{-}(\nabla \mathbf{v}) \mathrm{d} x+\int_{\Omega^{+}} f^{+}(\nabla \mathbf{v}) \mathrm{d} x & \\
\quad+\int_{B} g\left(\hat{\nabla} \mathbf{v} \mid \frac{1}{\varepsilon} \frac{\partial \mathbf{v}}{\partial x_{3}}\right) \mathrm{d} x-L(\mathbf{v})-\varepsilon L^{s}(\mathbf{v}) & \text { if } \mathbf{v} \in W_{\Gamma_{0}}^{1, p}\left(\Omega, \mathbb{R}^{3}\right), \\
+\infty & \text { otherwise, }\end{cases}
\end{aligned}
$$

The compactness result below has to be compared with the a priori estimates of Proposition 1 and the conclusions of the Corollary 1 . The assertions are direct consequences of the coercivity condition in (1) and Poincaré's inequality.

Lemma 1. Let $\left(\mathbf{v}_{\varepsilon}\right)_{\varepsilon>0} \in L^{p}\left(\Omega, \mathbb{R}^{3}\right)$ such that $\sup _{\varepsilon>0} \mathcal{F}_{\varepsilon}\left(\mathbf{v}_{\varepsilon}\right)<+\infty$, then

(i) there exist $\mathbf{v} \in W_{\Gamma_{0}}^{1, p}\left(\Omega, \mathbb{R}^{3}\right), \mathbf{w} \in L^{p}\left(B, \mathbb{R}^{3}\right)$ and a subsequence of $\left(\mathbf{v}_{\varepsilon}\right)_{\varepsilon>0}$ not relabeled such that $\mathbf{v}_{\varepsilon} \rightarrow \mathbf{v}$ weakly in $W_{\Gamma_{0}}^{1, p}\left(\Omega, \mathbb{R}^{3}\right)$ and strongly in $L^{p}\left(\Omega, \mathbb{R}^{3}\right)$;

(ii) $\frac{\partial \mathbf{v}_{\varepsilon}}{\partial x_{3}} \rightarrow 0$ strongly in $L^{p}\left(B, \mathbb{R}^{3}\right)$ and so $\frac{\partial \mathbf{v}}{\partial x_{3}}=0$ in $B$;

(iii) $\frac{1}{\varepsilon} \frac{\partial \mathbf{v}_{\varepsilon}}{\partial x_{3}} \rightarrow \mathbf{w}$ weakly in $L^{p}\left(B, \mathbb{R}^{3}\right)$.

Let us consider the functional $\mathcal{F}: L^{p}\left(\Omega, \mathbb{R}^{3}\right) \rightarrow \mathbb{R} \cup\{+\infty\}$ defined by

$$
\mathcal{F}(\mathbf{v}):=\left\{\begin{aligned}
& \int_{\Omega^{-}} \mathcal{Q} f^{-}(\nabla \mathbf{v}) \mathrm{d} x+\int_{\Omega^{+}} \mathcal{Q} f^{+}(\nabla \mathbf{v}) \mathrm{d} x \\
& \quad+\int_{B} \mathcal{Q} g_{0}(\hat{\nabla} \mathbf{v}) \mathrm{d} x-L(\mathbf{v}) \text { if } \mathbf{v} \in V_{B}^{p} \\
&+\infty \text { otherwise }
\end{aligned}\right.
$$

where $V_{B}^{p}=\left\{\mathbf{v} \in W_{\Gamma_{0}}^{1, p}\left(\Omega, \mathbb{R}^{3}\right) ; v_{i, 3}=0\right.$ in $\left.B\right\}$ and $\mathcal{Q} f^{ \pm}, \mathcal{Q} g_{0}$ denote the quasiconvexifications of $f^{ \pm}$ and $g_{0}$, and $g_{0}$ is defined for every $\hat{\lambda} \in \mathbf{M}^{3 \times 2}$ by $g_{0}(\hat{\lambda}):=\inf _{\xi \in \mathbb{R}^{3}} g(\hat{\lambda} \mid \xi)$; compare with (11) and (12).

The main result of this section is the following extension of Theorem 1.

Theorem 3. Let us equip the space $L^{p}\left(\Omega, \mathbb{R}^{3}\right)$ with the strong topology, then the sequence $\left(\mathcal{F}_{\varepsilon}\right)_{\varepsilon>0}$ $\Gamma$-converges to the functional $\mathcal{F}$.

The proof consists in establishing Propositions 5 and 6.

Proposition 5. For all $\mathbf{v} \in L^{p}\left(\Omega, \mathbb{R}^{3}\right)$ and all sequence $\left(\mathbf{v}_{\varepsilon}\right)_{\varepsilon>0}$ strongly converging to $\mathbf{v}$ in $L^{p}\left(\Omega, \mathbb{R}^{3}\right)$ the following inequality holds:

$$
\mathcal{F}(\mathbf{v}) \leqslant \liminf _{\varepsilon \rightarrow 0} \mathcal{F}_{\varepsilon}\left(\mathbf{v}_{\varepsilon}\right)
$$


Proof. Clearly one may assume that $\liminf _{\varepsilon \rightarrow 0} \mathcal{F}_{\varepsilon}\left(\mathbf{v}_{\varepsilon}\right)<+\infty$ so that from Lemma $1 \mathbf{v}$ belongs to $W_{\Gamma_{0}}^{1, p}\left(\Omega, \mathbb{R}^{3}\right)$ and $\frac{\partial \mathbf{v}}{\partial x_{3}}=0$ in $B$. Furthermore $\left(\mathbf{v}_{\varepsilon}\right)_{\varepsilon>0}$ weakly converges to $\mathbf{v}$ in $W_{\Gamma_{0}}^{1, p}\left(\Omega, \mathbb{R}^{3}\right)$. This prove that $\mathbf{v} \in V_{B}^{p}$. On the other hand one has

$$
\begin{aligned}
& \liminf _{\varepsilon \rightarrow 0} \mathcal{F}_{\varepsilon}\left(\mathbf{v}_{\varepsilon}\right) \\
& =\liminf _{\varepsilon \rightarrow 0}\left(\int_{\Omega^{-}} f^{-}\left(\nabla \mathbf{v}_{\varepsilon}\right) \mathrm{d} x+\int_{\Omega^{+}} f^{+}\left(\nabla \mathbf{v}_{\varepsilon}\right) \mathrm{d} x+\int_{B} g\left(\hat{\nabla} \mathbf{v}_{\varepsilon} \mid \frac{1}{\varepsilon} \frac{\partial \mathbf{v}_{\varepsilon}}{\partial x_{3}}\right) \mathrm{d} x-L\left(\mathbf{v}_{\varepsilon}\right)-\varepsilon L^{s}\left(\mathbf{v}_{\varepsilon}\right)\right) \\
& \geqslant \liminf _{\varepsilon \rightarrow 0} \int_{\Omega^{-}} f^{-}\left(\nabla \mathbf{v}_{\varepsilon}\right) \mathrm{d} x+\liminf _{\varepsilon \rightarrow 0} \int_{\Omega^{+}} f^{+}\left(\nabla \mathbf{v}_{\varepsilon}\right) \mathrm{d} x+\liminf _{\varepsilon \rightarrow 0} \int_{B} g\left(\hat{\nabla} \mathbf{v}_{\varepsilon} \mid \frac{1}{\varepsilon} \frac{\partial \mathbf{v}_{\varepsilon}}{\partial x_{3}}\right) \mathrm{d} x-L(\mathbf{v}) \\
& \geqslant \liminf _{\varepsilon \rightarrow 0} \int_{\Omega^{-}} \mathcal{Q} f^{-}\left(\nabla \mathbf{v}_{\varepsilon}\right) \mathrm{d} x+\liminf _{\varepsilon \rightarrow 0} \int_{\Omega^{+}} \mathcal{Q} f^{+}\left(\nabla \mathbf{v}_{\varepsilon}\right) \mathrm{d} x \\
& \quad+\liminf _{\varepsilon \rightarrow 0} \int_{B} g\left(\hat{\nabla} \mathbf{v}_{\varepsilon} \mid \frac{1}{\varepsilon} \frac{\partial \mathbf{v}_{\varepsilon}}{\partial x_{3}}\right) \mathrm{d} x-L(\mathbf{v}) .
\end{aligned}
$$

According to the classical membrane theory (see $[4,13]$ ) it is well known that

$$
\liminf _{\varepsilon \rightarrow 0} \int_{B} g\left(\hat{\nabla} \mathbf{v}_{\varepsilon} \mid \frac{1}{\varepsilon} \frac{\partial \mathbf{v}_{\varepsilon}}{\partial x_{3}}\right) \mathrm{d} x \geqslant \int_{B} \mathcal{Q} g_{0}(\hat{\nabla} \mathbf{v}) \mathrm{d} \hat{x} .
$$

We complete the proof by combining (20), the lsc property of integral functional with quasiconvex integrands and (21).

In the sequel, we need the following lemma.

Lemma 2. Let us consider the functional $\widetilde{\mathcal{F}}: L^{p}\left(\Omega, \mathbb{R}^{3}\right) \rightarrow \mathbb{R} \cup\{+\infty\}$ defined by

$$
\widetilde{\mathcal{F}}(\mathbf{v}):=\left\{\begin{array}{cl}
\int_{\Omega^{-}} f^{-}(\nabla \mathbf{v}) \mathrm{d} x+\int_{\Omega^{+}} f^{+}(\nabla \mathbf{v}) \mathrm{d} x \\
\quad+\int_{B} g_{0}(\hat{\nabla} \mathbf{v}) \mathrm{d} x-L(\mathbf{v}) & \text { if } \mathbf{v} \in V_{B}^{p}, \\
+\infty & \text { otherwise. }
\end{array}\right.
$$

Then the functional $\mathcal{F}$ is the lsc regularization of $\widetilde{\mathcal{F}}$ for the strong topology of $L^{p}\left(\Omega, \mathbb{R}^{3}\right)$.

Proof. Let us still denote the set $\Omega^{+} \cup S \cup \Omega^{-}$by $\Omega$ and by $\gamma_{S}$ the trace operator from $W^{1, p}(\Omega \backslash$ $\left.S, \mathbb{R}^{3}\right)$ into $L^{p}\left(S, \mathbb{R}^{3}\right)$. It is straightforward to establish that the spaces $V_{B}^{p}$ and $V^{p}:=\{\mathbf{v} \in$ $\left.W_{\Gamma_{0}}^{1, p}\left(\Omega, \mathbb{R}^{3}\right): \gamma_{S}(\mathbf{v}) \in W^{1, p}\left(S, \mathbb{R}^{3}\right)\right\}$ are isometric. Then setting $f=f^{+} 1_{\Omega^{+}}+f^{-} 1_{\Omega^{-}}$the functional $\widetilde{\mathcal{F}}$ may also be expressed as follows:

$$
\widetilde{\mathcal{F}}(\mathbf{v}):= \begin{cases}\int_{\Omega} f(\nabla \mathbf{v}) \mathrm{d} x+h \int_{S} g_{0}\left(\hat{\nabla} \gamma_{S}(\mathbf{v})\right) \mathrm{d} \hat{x}-L(\mathbf{v}) & \text { if } \mathbf{v} \in V^{p}, \\ \text { otherwise. }\end{cases}
$$

In the proof below we use this last formulation. We must establish

$$
\mathcal{F}(\mathbf{v})=\inf \left\{\liminf _{n \rightarrow+\infty} \tilde{\mathcal{F}}\left(\mathbf{v}_{n}\right): \mathbf{v}_{n} \rightarrow \mathbf{v} \in L^{p}\left(\Omega, \mathbb{R}^{3}\right)\right\}
$$


or equivalently, for all $\mathbf{v}$ in $L^{p}\left(\Omega, \mathbb{R}^{3}\right)$, the two following assertions:

$$
\begin{aligned}
& \text { for all } \mathbf{v}_{n} \rightarrow \mathbf{v} \text { in } L^{p}\left(\Omega, \mathbb{R}^{3}\right) \quad \liminf _{n \rightarrow+\infty} \tilde{\mathcal{F}}\left(\mathbf{v}_{n}\right) \geqslant \mathcal{F}(\mathbf{v}), \\
& \exists \mathbf{v}_{n} \in L^{p}\left(\Omega, \mathbb{R}^{3}\right) \text { s.t. } \quad \limsup _{n \rightarrow+\infty} \tilde{\mathcal{F}}\left(\mathbf{v}_{n}\right) \leqslant \mathcal{F}(\mathbf{v}) .
\end{aligned}
$$

Since $\mathbf{v} \mapsto L(\mathbf{v})$ is continuous for the strong convergence in $L^{p}\left(\Omega, \mathbb{R}^{3}\right)$ it suffices to establish the previous assertions by neglecting $L$ in the definition of $\mathcal{F}$ and $\tilde{\mathcal{F}}$.

Let $\mathbf{v}_{n} \rightarrow \mathbf{v}$ in $L^{p}\left(\Omega, \mathbb{R}^{3}\right)$ such that $\liminf _{n \rightarrow+\infty} \tilde{\mathcal{F}}\left(\mathbf{v}_{n}\right)<+\infty$. Then $\mathbf{v}_{n} \in W_{\Gamma_{0}}^{1, p}\left(\Omega, \mathbb{R}^{3}\right)$ and $\gamma_{S}\left(\mathbf{v}_{n}\right) \in W^{1, p}\left(S, \mathbb{R}^{3}\right)$. From the coercivity of $f$ and $g_{0}$ (the coercivity of $g_{0}$ is a straightforward consequence of that of $g), \mathbf{v}_{n}$ weakly converges to $\mathbf{v}$ in $W_{\Gamma_{0}}^{1, p}\left(\Omega, \mathbb{R}^{3}\right)$ and $\gamma_{S}\left(\mathbf{v}_{n}\right)$ weakly converges to $\gamma_{S}(\mathbf{v})$ in $W^{1, p}\left(S, \mathbb{R}^{3}\right)$. Consequently

$$
\begin{aligned}
\liminf _{n \rightarrow+\infty} \tilde{\mathcal{F}}\left(\mathbf{v}_{n}\right) & \geqslant \liminf _{n \rightarrow+\infty} \int_{\Omega} f\left(\nabla \mathbf{v}_{n}\right) \mathrm{d} x+\liminf _{n \rightarrow+\infty} \int_{S} g_{0}\left(\hat{\nabla} \gamma_{S}\left(\mathbf{v}_{n}\right)\right) \mathrm{d} \hat{x} \\
& \geqslant \liminf _{n \rightarrow+\infty} \int_{\Omega} \mathcal{Q} f\left(\nabla \mathbf{v}_{n}\right) \mathrm{d} x+\liminf _{n \rightarrow+\infty} \int_{S} \mathcal{Q} g_{0}\left(\hat{\nabla} \gamma_{S}\left(\mathbf{v}_{\mathbf{n}}\right)\right) \mathrm{d} \hat{x} \\
& \geqslant \mathcal{F}(\mathbf{v}) .
\end{aligned}
$$

We have used the weak lower semicontinuity in $W_{\Gamma_{0}}^{1, p}\left(\Omega, \mathbb{R}^{3}\right)$ and $W^{1, p}\left(S, \mathbb{R}^{3}\right)$ of the two integral functionals to pass from the second to the third line.

On the other hand, let $\mathbf{v}$ in $L^{p}\left(\Omega, \mathbb{R}^{3}\right)$ such that $\mathcal{F}(\mathbf{v})<+\infty$ and consider a sequence $\left(\left(\mathbf{u}_{n}, \mathbf{v}_{n}\right)\right)_{n \in \mathbb{N}}$ of functions in $W_{\Gamma_{0}}^{1, p}\left(\Omega, \mathbb{R}^{3}\right) \times W^{1, p}\left(S, \mathbb{R}^{3}\right)$ weakly converging to $\left(\mathbf{v}, \gamma_{S}(\mathbf{v})\right)$ in $W_{\Gamma_{0}}^{1, p}\left(\Omega, \mathbb{R}^{3}\right) \times W^{1, p}\left(S, \mathbb{R}^{3}\right)$ and strongly in $L^{p}\left(\Omega, \mathbb{R}^{3}\right) \times L^{p}\left(S, \mathbb{R}^{3}\right)$ such that

$$
\begin{aligned}
& \lim _{n \rightarrow+\infty} \int_{\Omega} f\left(\nabla \mathbf{u}_{n}\right) \mathrm{d} x=\int_{\Omega} \mathcal{Q} f(\nabla \mathbf{v}) \mathrm{d} x, \\
& \lim _{n \rightarrow+\infty} \int_{S} g_{0}\left(\hat{\nabla} \mathbf{v}_{n}\right) \mathrm{d} \hat{x}=\int_{S} \mathcal{Q} g_{0}\left(\hat{\nabla} \gamma_{S}(\mathbf{v})\right) \mathrm{d} \hat{x} .
\end{aligned}
$$

Such sequences exist, consult for instance [4], Theorem 11.2.1. Note that we cannot claim that $\gamma_{S}\left(\mathbf{u}_{n}\right)=\mathbf{v}_{n}$. We are going to modify the function $\mathbf{u}_{n}$ near $S$ so that this constraint holds, i.e. in order that the new function $\tilde{\mathbf{v}}_{n}$ belongs to $V^{p}$. In what follows, the functions $\mathbf{v}_{n}$ and $\gamma_{S}(\mathbf{v})$ will be indifferently considered as functions in $W^{1, p}\left(S, \mathbb{R}^{3}\right)$ or in $W^{1, p}\left(\Omega, \mathbb{R}^{3}\right)$. By coercivity of $g_{0}, \int_{S}\left|\hat{\nabla} \mathbf{v}_{n}\right|^{p} \mathrm{~d} \hat{x}$ is bounded, thus, for every $m \in \mathbb{N}^{*}$ one can choose $\eta>0$ small enough so that in the layer $\Sigma_{\eta}:=S \times(-\eta, \eta)$ the two following inequalities hold:

$$
\begin{aligned}
& \int_{\Sigma_{\eta}}\left(1+\left|\hat{\nabla} \mathbf{v}_{n}\right|^{p}\right) \mathrm{d} x=2 \eta\left(\mathcal{H}^{2}(S)+\int_{S}\left|\hat{\nabla} v_{n}\right|^{p} \mathrm{~d} \hat{x}\right) \leqslant \frac{1}{m}, \\
& \int_{\Sigma_{\eta}}\left|\frac{\partial v}{\partial x_{3}}\right|^{p} \mathrm{~d} x \leqslant \frac{1}{m^{p}} .
\end{aligned}
$$

The idea is now to modify $\mathbf{u}_{n}$ on $\Sigma_{\eta}$ in order that the trace on $S$ of the new function $\tilde{\mathbf{v}}_{n}$ be equal to $\mathbf{v}_{n}$, and in such a way to decrease $\lim _{n \rightarrow+\infty} \int_{\Omega} f\left(\nabla \mathbf{u}_{n}\right) \mathrm{d} x$. We use the slicing method of De Giorgi. Let 
us slice the layer $\Sigma_{\eta}$ as follows: for $i=1, \ldots, m$, consider $\left.\Sigma_{i}:=S \times\right]-i \frac{\eta}{m}, i \frac{\eta}{m}\left[\right.$ and $\left(\Omega_{i}\right)_{i=1, \ldots, m}$ the decreasing sequence of open subsets $\Omega_{i}:=\Omega \backslash \Sigma_{i}$. With the convention $\Sigma_{0}=\emptyset$ we have

$$
\begin{aligned}
\Sigma_{\eta} & =\bigcup_{i=0}^{m-1}\left(\Sigma_{i+1} \backslash \Sigma_{i}\right), \\
\Omega_{m} & =\Omega \backslash \Sigma_{\eta} \subset \subset \cdots \subset \subset \Omega_{i+1} \subset \subset \Omega_{i} \subset \cdots \subset \Omega_{1}=\Omega \backslash \Sigma_{\eta / m} \subset \Omega \backslash S .
\end{aligned}
$$

Note that $\Omega_{i} \backslash \Omega_{i+1}=\Sigma_{i+1} \backslash \Sigma_{i}$. Let $\left(\varphi_{i}\right)_{i=0, \ldots, m-1}$ be a sequence of functions in $\mathcal{C}^{1}\left(\mathbb{R}^{3}\right)$ satisfying

$$
\begin{aligned}
& \varphi_{i}=1 \quad \text { on } \Omega_{i+1}, \quad \varphi_{i}=0 \quad \text { on } \Sigma_{i}, 0 \leqslant \varphi_{i} \leqslant 1 \\
& \left|\nabla \varphi_{i}\right| \leqslant \frac{m}{\eta}
\end{aligned}
$$

and define

$$
\mathbf{u}_{n, i}=\varphi_{i}\left(\mathbf{u}_{n}-\mathbf{v}_{n}\right)+\mathbf{v}_{n}
$$

Clearly $\mathbf{u}_{n, i}$ belongs to $W_{\Gamma_{0}}^{1, p}\left(\Omega, \mathbb{R}^{3}\right)$ and $\gamma_{s}\left(\mathbf{u}_{n, i}\right)=\mathbf{v}_{n}$. For $i=0, \ldots, m-1$ we have

$$
\begin{aligned}
\int_{\Omega} f\left(\nabla \mathbf{u}_{n, i}\right) \mathrm{d} x & =\int_{\Omega \backslash \Omega_{i}} f\left(\nabla \mathbf{u}_{n, i}\right) \mathrm{d} x+\int_{\Omega_{i} \backslash \Omega_{i+1}} f\left(\nabla \mathbf{u}_{n, i}\right) \mathrm{d} x+\int_{\Omega_{i+1}} f\left(\nabla \mathbf{u}_{n, i}\right) \mathrm{d} x \\
& =\int_{\Sigma_{i}} f\left(\nabla \mathbf{v}_{n}\right) \mathrm{d} x+\int_{\Sigma_{i+1} \backslash \Sigma_{i}} f\left(\nabla \mathbf{u}_{n, i}\right) \mathrm{d} x+\int_{\Omega_{i+1}} f\left(\nabla \mathbf{u}_{n}\right) \mathrm{d} x \\
& \leqslant \int_{\Sigma_{\eta}} f\left(\nabla \mathbf{v}_{n}\right) \mathrm{d} x+\int_{\Sigma_{i+1} \backslash \Sigma_{i}} f\left(\nabla \mathbf{u}_{n, i}\right) \mathrm{d} x+\int_{\Omega} f\left(\nabla \mathbf{u}_{n}\right) \mathrm{d} x
\end{aligned}
$$

Then, from (24) and the growth condition in (1), we obtain

$$
\begin{aligned}
\int_{\Omega} f\left(\nabla \mathbf{u}_{n, i}\right) \mathrm{d} x \leqslant & C\left(\frac{1}{m}+\left(\frac{m}{\eta}\right)^{p} \int_{\Sigma_{i+1} \backslash \Sigma_{i}}\left|\mathbf{u}_{n}-\mathbf{v}_{n}\right|^{p} \mathrm{~d} x+\int_{\Sigma_{i+1} \backslash \Sigma_{i}}\left(\left|\nabla \mathbf{u}_{n}\right|^{p}\right) \mathrm{d} x\right) \\
& +\int_{\Omega} f\left(\nabla \mathbf{u}_{n}\right) \mathrm{d} x,
\end{aligned}
$$

where, from now on, $C$ denotes various positive constants depending only on $\beta, p$ and $\Omega$. By averaging these $m$ inequalities, we obtain

$$
\begin{aligned}
\frac{1}{m} \sum_{i=0}^{m-1} \int_{\Omega} f\left(\nabla \mathbf{u}_{n, i}\right) \mathrm{d} x \leqslant & C\left(\frac{1}{m}+\frac{1}{m}\left(\frac{m}{\eta}\right)^{p} \int_{\Sigma_{\eta}}\left|\mathbf{u}_{n}-\mathbf{v}_{n}\right|^{p} \mathrm{~d} x+\frac{1}{m} \int_{\Sigma_{\eta}}\left|\nabla \mathbf{u}_{n}\right|^{p} \mathrm{~d} x\right) \\
& +\int_{\Omega} f\left(\nabla \mathbf{u}_{n}\right) \mathrm{d} x .
\end{aligned}
$$


We have used a slicing method in order to control the term $\int_{\Sigma_{\eta}}\left|\nabla \mathbf{u}_{n}\right|^{p} \mathrm{~d} x$ thanks to the coefficient $\frac{1}{m}$, $m \rightarrow+\infty$ (i.e. by taking the slices more and more thin). We could not conclude by using a simple truncation. Indeed from the coercivity condition in (1), $\int_{\Omega}\left|\nabla \mathbf{u}_{n}\right|^{p} \mathrm{~d} x$ is bounded, hence

$$
\frac{1}{m} \int_{\Sigma_{\eta}}\left|\nabla \mathbf{u}_{n}\right|^{p} \mathrm{~d} x \leqslant \frac{C}{m}
$$

so that

$$
\frac{1}{m} \sum_{i=0}^{m-1} \int_{\Omega} f\left(\nabla \mathbf{u}_{n, i}\right) \mathrm{d} x \leqslant C\left(\frac{1}{m}+\frac{1}{m}\left(\frac{m}{\eta}\right)^{p} \int_{\Sigma_{\eta}}\left|\mathbf{u}_{n}-\mathbf{v}_{n}\right|^{p} \mathrm{~d} x\right)+\int_{\Omega} f\left(\nabla \mathbf{u}_{n}\right) \mathrm{d} x .
$$

Let $i(n, m)$ be the index $i$ such that

$$
\int_{\Omega} f\left(\nabla \mathbf{u}_{n, i(n, m)}\right) \mathrm{d} x=\min _{i=0, \ldots, m-1} \int_{\Omega} f\left(\nabla \mathbf{u}_{n, i}\right) \mathrm{d} x .
$$

Inequality (26) then yields

$$
\int_{\Omega} f\left(\nabla \mathbf{u}_{n, i(n, m)}\right) \mathrm{d} x \leqslant C\left(\frac{1}{m}+\frac{1}{m}\left(\frac{m}{\eta}\right)^{p} \int_{\Sigma_{\eta}}\left|\mathbf{u}_{n}-\mathbf{v}_{n}\right|^{p} \mathrm{~d} x\right)+\int_{\Omega} f\left(\nabla \mathbf{u}_{n}\right) \mathrm{d} x,
$$

thus, from (23)

$$
\limsup _{n \rightarrow+\infty} \int_{\Omega} f\left(\nabla \mathbf{u}_{n, i(n, m)}\right) \mathrm{d} x \leqslant C\left(\frac{1}{m}+\frac{1}{m}\left(\frac{m}{\eta}\right)^{p} \int_{\Sigma_{\eta}}\left|\mathbf{v}-\gamma_{S}(\mathbf{v})\right|^{p} \mathrm{~d} x\right)+\int_{\Omega} \mathcal{Q} f(\nabla \mathbf{v}) \mathrm{d} x
$$

Clearly the following Poincaré inequality holds

$$
\int_{\Sigma_{\eta}}\left|\mathbf{v}-\gamma_{S}(\mathbf{v})\right|^{p} \mathrm{~d} x \leqslant \eta^{p} \int_{\Sigma_{\eta}}\left|\frac{\partial v}{\partial x_{3}}\right|^{p} \mathrm{~d} x
$$

and, combining this inequality with the second one in (24), (27) gives

$$
\limsup _{n \rightarrow+\infty} \int_{\Omega} f\left(\nabla \mathbf{u}_{n, i(n, m)}\right) \mathrm{d} x \leqslant C \frac{1}{m}+\int_{\Omega} \mathcal{Q} f(\nabla \mathbf{v}) \mathrm{d} x
$$

and finally, from (23)

$$
\limsup _{m \rightarrow+\infty} \limsup _{n \rightarrow \infty} \mathcal{F}\left(\mathbf{u}_{n, i(n, m)}\right) \leqslant \mathcal{F}(\mathbf{v}) .
$$

We conclude by a classical diagonalization argument: there exists $n \mapsto m(n)$ mapping $\mathbb{N}$ into $\mathbb{N}$ such that $m(n) \rightarrow+\infty$ when $n \rightarrow+\infty$ and

$$
\limsup _{n \rightarrow+\infty} \mathcal{F}\left(\mathbf{u}_{n, i(n, m(n))}, v_{n}\right) \leqslant \mathcal{F}(\mathbf{v})
$$


It remains to show that the sequence $\left(\tilde{\mathbf{v}}_{n}\right)_{n \in \mathbb{N}}$ defined by $\tilde{\mathbf{v}}_{n}=\mathbf{u}_{n, i(n, m(n))}$ strongly converges to $\mathbf{v}$ in $L^{p}\left(\Omega, \mathbb{R}^{3}\right)$. Let $\eta(n)$ be the value of $\eta$ corresponding to $m(n)$. It follows from (25) that

$$
\begin{aligned}
\int_{\Omega}\left|\tilde{\mathbf{v}}_{n}-\mathbf{v}\right|^{p} \mathrm{~d} x & =\int_{\Omega}\left|\left(1-\varphi_{i(n, m(n))}\right)\left(\mathbf{v}_{n}-\mathbf{u}_{n}\right)+\mathbf{u}_{n}-\mathbf{v}\right|^{p} \mathrm{~d} x \\
& \leqslant C\left(\int_{\Sigma_{i(n, m(n))+1}}\left|\mathbf{u}_{n}-\mathbf{v}_{n}\right|^{p} \mathrm{~d} x+\int_{\Omega}\left|\mathbf{u}_{n}-\mathbf{v}\right|^{p} \mathrm{~d} x\right) \\
& \leqslant C\left(\int_{\Sigma_{\eta(n)}}\left|\mathbf{u}_{n}-\mathbf{v}_{n}\right|^{p} \mathrm{~d} x+\int_{\Omega}\left|\mathbf{u}_{n}-\mathbf{v}\right|^{p} \mathrm{~d} x\right)
\end{aligned}
$$

and the claim follows easily since $\mathbf{u}_{n} \rightarrow \mathbf{v}$ strongly in $L^{p}\left(\Omega, \mathbb{R}^{3}\right), \mathbf{v}_{n} \rightarrow \gamma_{S}(\mathbf{v})$ strongly in $L^{p}\left(S, \mathbb{R}^{3}\right)$ and $\eta(n) \rightarrow 0$ when $n \rightarrow 0$ (for the first integral in the right-hand side write $\mathbf{u}_{n}-\mathbf{v}_{n}=\mathbf{v}-\gamma_{S}(\mathbf{v})+$ $\left.\left(\mathbf{u}_{n}-\mathbf{v}\right)+\left(\gamma_{S}(\mathbf{v})-\mathbf{v}_{n}\right)\right)$.

Proposition 6. The following inequality holds in $L^{p}\left(\Omega, \mathbb{R}^{3}\right)$

$$
\Gamma-\lim \sup \mathcal{F}_{\varepsilon}(\mathbf{v}) \leqslant \mathcal{F}(\mathbf{v})
$$

Proof. Let $\mathbf{v}$ be a fixed element in $L^{p}\left(\Omega, \mathbb{R}^{3}\right)$. One may assume $\mathcal{F}(\mathbf{v})<+\infty$ otherwise there is nothing to prove. Hence we can assume that $\mathbf{v} \in V_{B}^{p}$. According to a well-known result of interchange between infimum and integrals (see [3]), we have

$$
\int_{B} g_{0}(\hat{\nabla} \mathbf{v}) \mathrm{d} x=h \int_{S} g_{0}(\hat{\nabla} \mathbf{v}) \mathrm{d} \hat{x}=h \inf _{\boldsymbol{\xi} \in \mathcal{D}\left(S, \mathbb{R}^{3}\right)} \int_{S} g(\hat{\nabla} \mathbf{v} \mid \boldsymbol{\xi}) \mathrm{d} \hat{x} .
$$

Let $\xi \in \mathcal{D}\left(\Omega, \mathbb{R}^{3}\right)$, set $\phi(\mathbf{x})=x_{3} \boldsymbol{\xi}(\hat{x})$ for $\mathbf{x} \in B$, and define $\mathbf{v}_{\varepsilon}$ in $W_{\Gamma_{0}}^{1, p}\left(\Omega, \mathbb{R}^{3}\right)$ by:

$$
\mathbf{v}_{\varepsilon}(\mathbf{x}):=\mathbf{v}(\mathbf{x})+\varepsilon \phi(\mathbf{x})
$$

Then the sequence $\left(\mathbf{v}_{\varepsilon}\right)_{\varepsilon>0}$, strongly converges to $\mathbf{v}$ in $W_{\Gamma_{0}}^{1, p}\left(\Omega, \mathbb{R}^{3}\right)$ when $\varepsilon \rightarrow 0$. We have

$$
\begin{aligned}
\mathcal{F}_{\varepsilon}\left(\mathbf{v}_{\varepsilon}\right)= & \int_{\Omega^{-}} f^{-}\left(\nabla \mathbf{v}_{\varepsilon}\right) \mathrm{d} x+\int_{\Omega^{+}} f^{+}\left(\nabla \mathbf{v}_{\varepsilon}\right) \mathrm{d} x+\int_{B} g\left(\hat{\nabla} \mathbf{v}_{\varepsilon} \mid \frac{1}{\varepsilon} \frac{\partial \mathbf{v}_{\varepsilon}}{\partial x_{3}}\right) \mathrm{d} x-L\left(\mathbf{v}_{\varepsilon}\right)-\varepsilon L^{s}\left(\mathbf{v}_{\varepsilon}\right) \\
= & \int_{\Omega^{-}} f^{-}(\nabla \mathbf{v}+\varepsilon \nabla \phi) \mathrm{d} x+\int_{\Omega^{+}} f^{+}(\nabla \mathbf{v}+\varepsilon \nabla \boldsymbol{\phi}) \mathrm{d} x \\
& +\int_{B} g\left(\hat{\nabla} \mathbf{v}+\varepsilon x_{3} \hat{\nabla} \boldsymbol{\xi} \mid \boldsymbol{\xi}\right) \mathrm{d} x-L\left(\mathbf{v}_{\varepsilon}\right)-\varepsilon L^{s}\left(\mathbf{v}_{\varepsilon}\right),
\end{aligned}
$$

hence

$$
\lim _{\varepsilon \rightarrow 0} \mathcal{F}_{\varepsilon}\left(\mathbf{v}_{\varepsilon}\right)=\int_{\Omega^{-}} f^{-}(\nabla \mathbf{v}) \mathrm{d} x+\int_{\Omega^{+}} f^{+}(\nabla \mathbf{v}) \mathrm{d} x+h \int_{S} g(\hat{\nabla} \mathbf{v} \mid \boldsymbol{\xi}) \mathrm{d} \hat{x}-L(\mathbf{v}) .
$$


Taking the infimum over all $\xi \in \mathcal{D}\left(S, \mathbb{R}^{3}\right)$ in (31), from (29) we deduce

$$
\inf \left\{\limsup _{\varepsilon \rightarrow 0} \mathcal{F}_{\varepsilon}\left(\mathbf{v}_{\varepsilon}\right): \mathbf{v}_{\varepsilon} \rightarrow \mathbf{v} \text { in } L^{p}\left(\Omega, \mathbb{R}^{3}\right)\right\} \leqslant \tilde{\mathcal{F}}(\mathbf{v})
$$

i.e. $\Gamma-\lim \sup \mathcal{F}_{\varepsilon} \leqslant \tilde{\mathcal{F}}$ where the functional $\tilde{\mathcal{F}}$ is defined in (22). Taking the lower semicontinuous envelope of both functionals for the strong topology of $L^{p}\left(\Omega, \mathbb{R}^{3}\right)$, the conclusion then follows from the lower semicontinuity of $\Gamma-\lim \sup \mathcal{F}_{\varepsilon}$ and from Lemma 2.

\section{Concluding remarks}

1. The introduction of the transmission conditions of Ventcel type (in the linear case) seems new. Ventcel boundary conditions have been introduced by Lemrabet [14,15] who also studied the regularity of the solution when the domain is of polyhedral (or polygonal) type.

2. In the linear and scalar case, one can prove that a suitable decomposition domain algorithm converges. For this, the problem is reduced to an integral operator whose spectral properties allow to prove the convergence of a GMRES algorithm, [5].

3. When the atlas used for the surface $S$ needs more than one chart, for instance when the surface $S$ is a closed surface surrounding an inclusion one has to adapt the method of [6], i.e. to write the energy functional in terms of two arguments, one $\mathbf{u}$, the displacement on $\Omega_{\varepsilon}$, the other $\mathbf{v}$, the displacement on $B_{\varepsilon}$ occupied by the strong material.

4. One can consider a more general situation, with many layers of this type of multimaterial. When the number of layers increases, a homogenization method could be coupled with the previous analysis.

5. In [6] we find the non linear formulation of Section 4 as a macroscopic version of a new model taking into account gradient oscillations.

\section{References}

[1] E. Acerbi, G. Buttazzo and D. Percivale, Thin inclusions in linear elasticity: a variational approach, J. Reine Angew. Math. 386 (1988), 99-115.

[2] O. Anza Hafsa and J.P. Mandallena, The nonlinear membrane energy: variational derivation under the constraint $\operatorname{det} \nabla u>0$, Bulletin des Sciences Mathtématiques, to appear.

[3] O. Anza Hafsa and J.P. Mandallena, Interchange of infimum and integral, Calc. Var. 18 (2003), $433-449$.

[4] H. Attouch, G. Buttazzo and G. Michaille, Variational Analysis in Sobolev and BV Spaces: Application to PDEs and Optimization, MPS-SIAM Book Series on Optimization, Vol. 6, SIAM, Philadelphia, PA, 2005.

[5] A.L. Bessoud and F. Krasucki, GMRES algorithm for multi-materials with strong interface, C. R. Acad. Sci. Paris, Ser. 1 343 (2006), 297-282.

[6] A.L. Bessoud, F. Krasucki and G. Michaille, A relaxation procedure for bifunctionals of displacement - young measure state variables: a model of multi-material with micro-structured strong interface, submitted.

[7] H. Brezis, L.A. Caffarelli and A. Friedman, A reinforcement problem for elliptic equations and variational inequalities, Ann. Mat. Pura Appl. 4(123) (1980), 219-246.

[8] D. Caillerie, The effect of a thin inclusion of hight rigidity in an elastic body, Math. Meth. Appl. Sci. 2 (1980), 251-270.

[9] P.G. Ciarlet and P. Destuynder, A justification of the two-dimensional plate model, J. Mécanique 18 (1979) $315-344$.

[10] G. Geymonat and F. Krasucki, A limit problem of a soft thin joint, in: Partial Differential Equations and Applications, P. Marcellini, G. Talenti and E. Vesentini, eds, Marcel Dekker, New York, 1996, pp. 165-173.

[11] G. Geymonat, F. Krasucki and S. Lenci, Mathematical analysis of a bonded joint with a soft thin adhesive, Math. Mech. Solids 4 (1999), 201-255.

[12] M. Goland and E. Reissner, The stresses in cemented joints, J. Appl. Mech. ASME 11 (1944), A17-A27. 
[13] H. Le Dret and A. Raoult, The nonlinear membrane model as variational limit in nonlinear three-dimensional elasticity, J. Math. Pures Appl. Ser. IX 74 (1995), 549-578.

[14] K. Lemrabet, Etude de divers problème aux limites de Ventcel d'origine physique ou mécanique dans des domaines non réguliers. PhD thesis, Université Houari Boumediène d'Alger, 10 Mai 1987.

[15] K. Lemrabet, Problèmes de Ventcel pour le système de l'élasticité dans un domaine de $\mathbb{R}^{3}$, C. R. Acad. Sci. Paris, Sér. 1 Math. 304 (1987), 151-154.

[16] C. Licht and G. Michaille, A modelling of elastic adhesive bonding joints, Adv. Math. Sci. Appl. 7(2) (1997), 711-740.

[17] P. Suquet, Discontinuities and plasticity, in: Nonsmooth Mechanics and Application, J.J. Moreau and P.D. Panagiotopoulos, eds, CISM Courses and Lectures, Vol. 302, Springer-Verlag, Wien, 1988, pp. 280-340.

[18] A.D. Ventcel', On boundary conditions for multidimensional diffusion processes, Theory Probab. Appl. 4 (1959), 164177. 